\title{
Factors Influencing Postoperative Urinary Retention Following Elective Posterior Lumbar Spine Surgery: A Prospective Study
}

\author{
Siddharth Narasimhan Aiyer, Ajit Kumar, Ajoy Prasad Shetty, \\ Rishi Mugesh Kanna, Shanmuganath Rajasekaran \\ Department of Spine Surgery, Ganga Hospital, Coimbatore, India
}

\section{Study Design: Prospective observational study.}

Purpose: To determine the incidence of postoperative urinary retention (POUR) in patients undergoing elective posterior lumbar spine surgery and identify the risk factors associated with the development of POUR.

Overview of Literature: POUR following surgery can lead to detrusor dysfunction, urinary tract infections, prolonged hospital stay, and a higher treatment cost; however, the risk factors for POUR in spine surgery remain unclear.

Methods: A prospective, consecutive analysis was conducted on patients undergoing elective posterior lumbar surgery in the form of lumbar discectomy, lumbar decompression, and single-level lumbar fusions during a 6-month period. Patients with spine trauma, preoperative neurological deficit, previous urinary disturbance/symptoms, multiple-level fusion, and preoperative catheterization were excluded from the study. Potential patient- and surgery-dependent risk factors for the development of POUR were assessed. Univariate analysis and a multiple logistical regression analysis were performed.

Results: A total of 687 patients underwent posterior lumbar spine surgery during the study period; among these, 370 patients were included in the final analysis. Sixty-one patients developed POUR, with an incidence of $16.48 \%$. Significant risk factors for POUR were older age, higher body mass index (BMI), surgery duration, intraoperative fluid administration, lumbar fusion versus discectomy/ decompression, and higher postoperative pain scores ( $p<0.05$ for all). Sex, diabetes, and the type of inhalational agent used during anesthesia were not significantly associated with POUR. Multiple logistical regression analysis, including age, BMI, surgery duration, intraoperative fluid administration, fusion surgery, and postoperative pain scores demonstrated a predictive value of $92 \%$ for the study population and $97 \%$ for the POUR group.

Conclusions: POUR was associated with older age, higher BMI, longer surgery duration, a larger volume of intraoperative fluid administration, and higher postoperative pain scores. The contribution of postoperative pain scores in the multiple regression analysis was a significant predictor of POUR.

Keywords: Urinary retention; Postoperative complications; Spinal fusion; Urinary catheterization; Risk factors 


\section{Introduction}

Postoperative urinary retention (POUR) can result in bladder atony and increase the risk of urinary tract infection and sepsis [1]. Although several studies have been conducted on POUR, most of them are limited to general surgery, colorectal surgery, and total joint arthroplasty [27]. Few studies evaluated POUR in spine surgery, and a majority of those are retrospective case series [8-10]. Factors reported to have an association with the development of POUR include age, diabetes, opioid administration, anesthesia type, perioperative analgesia, intravenous (IV) fluid volume, comorbid medical and surgical conditions, and surgery duration $[1,6,11]$. The lack of accurate and complete data in a retrospective study makes the analysis suboptimal. The reported incidence of POUR ranges between $5 \%$ and $84 \%$, and numerous criteria have been suggested to define POUR, adding to the complexity of this clinical condition $[1,2,4,5,9]$. Studies have demonstrated that POUR with subsequent catheterization can result in urinary infection and bacteremia, which can be a source for surgical site wound infection and morbidity $[6,9,10,12-14]$. The identification of the risk factors for POUR may help reduce the chances of postoperative urinary infection, urinary bladder atony, and urosepsis, as well as reduce the length of hospital stay. We aimed to perform a prospective study to identify the patient- and surgical procedure-related risk factors for the development of POUR in micro-lumbar discectomy, lumbar decompression, and single-level fusions. These surgical procedures were considered owing to their shorter operative time and the fact that they may not require a routine preoperative urinary catheterization

\section{Materials and Methods}

A prospective consecutive recruitment was performed on patients undergoing elective posterior lumbar spine surgery performed between January 2015 and July 2015. The study was approved by the institutional review board of Ganga Hospital, Coimbatore, India (IRB approval no., 03/12/2014), and all ethical principles in the declaration of Helsinki were followed. And written informed consent was obtained from all participants. The study was performed at a single center with surgeries being performed by three surgeons.

\section{Inclusion and exclusion criteria}

Patients undergoing posterior elective lumbar spine surgery were included in the study. Patients scheduled for micro-lumbar discectomy, single- and multiple-level lumbar decompression, and single-level posterior lumbar fusions were identified and evaluated for the development of POUR. Multiple-level lumbar fusion, anterior lumbar surgery, acute spinal trauma, presence of preoperative neurological deficit, and a previous history of urinary disturbances were excluded from the study group. Patients were assessed clinically to identify any saddle anesthesia and sphincter function to determine possible bladder bowel dysfunction indicative of a cauda equina syndrome; such patients were excluded from the study. Any patients with significant preoperative neurological motor and sensory deficit (Medical Research Council [MRC] grade <3) were also excluded from the study because these patients may have a subclinical bladder deficit. Patients with features of urinary hesitancy, poor stream, nocturia, or on treatment for prostatic hypertrophy with alpha agonists were excluded from the study.

Patients who were unable to void urine postoperatively, with a lower suprapubic discomfort on palpation or inability to void with a palpable full bladder were defined as having POUR. Patients who did not develop any retention comprised the control group. No in-out catheterization was performed. All the patients were allowed to assume an erect posture to facilitate voiding; however, on failure to void, an indwelling urinary catheter was inserted. Catheter removal was attempted on the second postoperative day or once the patient was mobilized and ambulant, whichever occurred earlier.

Comparative analysis was performed between the POUR and control groups. Patient factors that were analyzed included age, sex, body mass index (BMI), and the presence of comorbidities, including diabetes mellitus and ischemic heart disease. The surgery-related factors that were assessed included postoperative pain scores (using Visual Analog Scale [VAS] scores), surgery duration, IV fluid volume administered, inhalational agent used (isoflurane or sevoflurane), and type of surgery performed (discectomy/decompression versus lumbar fusion).

All the surgeries were performed under general anesthesia with propofol as the induction agent, rocuronium as the muscle relaxant, $60 \mathrm{mcg}$ fentanyl as an opioid, and sevoflurane or isoflurane as the inhalational agent. Post- 
Table 1. Risk factors evaluated in the univariate analysis

\begin{tabular}{|c|c|c|c|}
\hline Characteristic & Postoperative urinary retention ( $\mathrm{N}=61$ ) & Control (N=309) & $p$-value \\
\hline Age (yr) & $49.7 \pm 14.1$ & $45.5 \pm 13.4$ & $0.036^{a)}$ \\
\hline Body mass index $\left(\mathrm{kg} / \mathrm{m}^{2}\right)$ & $25.6 \pm 2.3$ & $24.1 \pm 3.8$ & $<0.0001^{\text {a) }}$ \\
\hline Postoperative pain (Visual Analog Scale score) & $4.6 \pm 0.6$ & $2.9 \pm 1.2$ & $<0.0001^{\text {a) }}$ \\
\hline Duration of surgery (min) & $137 \pm 40.9$ & $89.4 \pm 34.8$ & $<0.0001^{\text {a) }}$ \\
\hline Intravenous fluids (mL) & $1,486 \pm 450$ & $1,268 \pm 381$ & $0.001^{\text {a) }}$ \\
\hline Hospital stay (day) & $5.13 \pm 1.19$ & $5.46 \pm 1.38$ & 0.06 \\
\hline Sex & & & 0.548 \\
\hline Male & 35 & 190 & \\
\hline Female & 26 & 119 & \\
\hline Diabetes & & & 0.066 \\
\hline Diabetic & 6 & 61 & \\
\hline Non-diabetic & 55 & 248 & \\
\hline Ischemic heart disease & 1 & 9 & 0.551 \\
\hline Surgery & & & $0.001^{\mathrm{al}}$ \\
\hline Decompression/discectomy & 27 & 209 & \\
\hline Fusion & 34 & 100 & \\
\hline
\end{tabular}

Values are presented as mean \pm standard deviation or number.

a)Denotes statistical significance.

operatively, all the patients received a single dose of $1 \mathrm{mg}$ butorphanol at night on the day of the surgery with no other opioid agent being used in the postoperative period. Postoperative analgesia was provided with non-steroidal anti-inflammatory drugs, paracetamol and rescue analgesia was provided with tramadol.

\section{Statistical analysis}

Univariate analysis was performed for continuous variables (age, BMI, surgery duration, amount of IV fluids injected, postoperative VAS score, and duration of hospital stay) using Student $t$-test, while categorical variables (sex, diabetes mellitus, ischemic heart disease, inhalation agent used, and type of surgery) were analyzed using chi-square statistical test (R ver. 2.15.0; https://www.r-project.org/) (Table 1). Statistical significance was set at $p<0.05$. Thereafter, the variables found to be significant in the univariate analysis were subjected to multiple regression analysis to identify the confounding factors and identify the best possible combination of variables that can help predict POUR.

\section{Results}

Total 687 patients underwent elective posterior spine surgery during the study period. After considering the inclusion and exclusion criteria, 370 patients, including those who underwent elective micro-lumbar discectomies, single- and multiple-level decompressions, and singlelevel posterior lumbar fusions were included for the final analysis. Eight patients presented with cauda equina with preoperative urinary dysfunction, and 23 patients had significant preoperative neurological motor and sensory deficit (MRC grade <3); these patients were excluded. Total 286 patients were excluded because of multiple-level fusion, spinal trauma, and previous history of urine voiding difficulties. Sixty-one of the 370 patients developed POUR, resulting in a prevalence rate of $16.5 \%$.

\section{Univariate analyses}

The study population comprised 225 men and 145 women. There were 26 women and 35 men in the POUR group, while the control group included 119 women and 190 men; there were not significant sex-based differences ( $p=0.549)$ with respect to the development of POUR. Six 
patients $(9.8 \%)$ in the POUR group were diabetic compared to $61(19.7 \%)$ in the control group; however, this difference did not reach statistical significance $(p=0.06)$. On comparing the use of the inhalational agent, isoflurane versus sevoflurane and the development of POUR, no significant difference was observed with $(p=0.273)$. The mean duration of stay in the POUR group $(5.13 \pm 1.19$ days) was not significantly different from that in the control group $(5.46 \pm 1.38$ days, $p=0.06)$.

In the POUR group, micro-lumbar discectomy and lumbar decompression were performed for 27 patients, and single-level fusion was performed for 34 patients. The control group had 209 (micro-lumbar discectomies+lumbar decompressions), and 100 lumbar fusions. Evaluation of discectomy/decompression versus fusion showed a significantly higher risk of POUR in the fusion group $(p=0.001)$. The details of the univariate analysis are listed in Table 1.

\section{Multiple logistical regression analyses}

Factors that were found significant in the univariate analysis were further assessed using multiple logistic regression analysis that also accounted for the confounding factors. Risk factors, such as age, BMI, duration of surgery, IV fluid volume, and fusion surgery became insignificant; while VAS score continued showing significance, suggesting that postoperative VAS score is independently capable of explaining most of the variation in POUR occurrence, and thus its prediction. When all these factors were included, regression produced an excellent overall predictive value of $92 \%$, with a value of $97 \%$ for the POUR group and $71 \%$ for the non-POUR groups. When the factor of VAS score was excluded, except for age, all the other factors remained significant. However, the overall prediction rate was reduced to $89 \%$, with a significant reduction in the value for the POUR (to 57\%) and an increase in the value for the non-POUR groups (to 96\%).

\section{Discussion}

Various authors have reported different prevalence rates for POUR, ranging from $5 \%$ to $84 \%[1,2,4,5,9]$. These variations can be attributed to the several factors, including the variable definitions used to define POUR, type of surgery performed, type of anesthesia, and use of opioid analgesia that affects bladder function [1-3,6-10,12].
Higher prevalence rates for POUR have been noted in patients who received spinal anesthesia, patient-controlled analgesia, and opioids as well as those who underwent anorectal surgery and total joint replacement surgery than in those who underwent other orthopedic surgical procedures $[1,2,6,12]$.Another contributing factor to the variable incidence rates is the fact that variable definitions have been used in these studies to establish POUR. In a review article, Baldini et al. [1] stated that the definition for POUR can be based on clinical, catheterization-, and ultrasonography-based evidence and documented over 18 different definitions used in previous studies [1]. The authors suggest that future studies focus on formulating and establishing a globally accepted definition for POUR.

The present study used a clinical definition for defining POUR, and this may be a limitation, particularly in the case of obese patients [15]. In a recent study, the use of ultrasonography-based identification for bladder volumes has been suggested as being superior to clinical assessment $[15,16]$. Moreover, patients with large bladder volumes and incomplete emptying may remain asymptomatic, and thus may escape detection based on a pure clinical definition [17]. This study did not perform a formal urological consult in all patients with POUR to rule out other asymptomatic and co-existing urinary problems, including benign prostatic hypertrophy $(\mathrm{BPH})$; this is another study limitation. However, all the patients in the POUR group were able to void normally after catheter removal, except one patient who needed prolonged catheterization.

POUR has been studied extensively in gastrointestinal and anorectal studies, in orthopedic surgery under spinal anesthesia, and most notably in total joint arthroplastyrelated procedures $[1-3,6,7,12]$. However, few studies have assessed POUR with lumbar spine surgery [8-10]. Another limitation is that the majority of the existing literature on POUR comprises retrospective reviews that are susceptible to bias [8-10].

In a large retrospective review on 15,681 patients who underwent major orthopedic surgery, including joint replacement surgery, Sung et al. [6] reported a prevalence rate of $2.3 \%$. Our prospective study documented a rate of $16.5 \%$ that is considerably higher than previous reports on POUR in spine surgery, such as that by Atschul et al. [8] (8.8\%), Gandhi et al. [9] (5.6\%), and Jung et al. [10] (11.1\%).

In-out catheterization has been associated with an increased cumulative risk of urinary tract infection owing 
to frequent manipulation of the lower urinary tract [12]. Therefore, we did not perform in-out catheterization, and an indwelling catheter was inserted for patients unable to void in the postoperative period. Lumbar discectomy/ decompression and single-level fusions were included because these patients are routinely discharged by the 3rd and 5th postoperative day respectively. Urinary catheterization is not performed in these procedures routinely, and the occurrence of POUR in this subset may prolong hospital stay. Patients undergoing multiple-level fusion and presenting with preoperative neurological deficit frequently require preoperative catheterization; thus, they were excluded from the prospective analysis.

General anesthesia, spinal anesthesia, opioids, and patient-controlled anesthesia are reported to cause POUR $[1,2,12]$. The authors speculate that postoperative pain is a subjective assessment and can be considerably influenced by the use of narcotics and patient-controlled analgesia devices in the postoperative period. The methods for postoperative analgesia were standardized to reduce the impact of narcotic use as an independent factor for POUR. All the procedures were performed under general anesthesia with uniform induction and maintenance protocols; further, the use of opioids in the postoperative period were restricted to a single dose to reduce the effect of these confounding factors. Postoperative analgesia was provided with non-steroidal anti-inflammatory drugs, Paracetamol and rescue analgesia was provided with Tramadol.

This study demonstrated that older age, higher BMI, and increased postoperative pain scores (VAS s cores) were the patient-related factors associated with higher risk of POUR. The documented surgical factors linked with increased risk of POUR were prolonged surgical duration, larger volume of IV fluids, and fusion surgery compared to discectomy and decompression.

Limited studies have examined POUR in spine surgery; Jung et al. [10] reported on 325 patients undergoing anterior cervical spine surgery for cervical radiculopathy and myelopathy. The authors concluded that older age, diabetes, male sex, BPH, clinical presentation of myelopathy, and narcotic drug use were associated with a higher risk of POUR [10]. They reported a $16 \%$ risk of infection and bladder complications in the 36 patients with POUR [10].

Gandhi et al. [9] conducted a retrospective analysis of POUR among 647 patients undergoing posterior lumbar spine surgery and reported that age, male sex, diabetes mellitus, and BPH were significant factors for POUR. However, the type of lumbar spine surgery, BMI, hypertension, and duration of surgery did not influence the occurrence of POUR [9]. This was in contrast to the present findings.

A major concern following POUR and urinary catheterization is the development of urinary tract infections. Wald et al. [13] analyzed 35,904 in patients undergoing major surgery, including cardiovascular, gastrointestinal surgery, and joint replacement surgery and concluded that urinary catheterization for more than 2 days increases the risk of urinary tract infection by two-fold. There were 61 patients in the POUR group with a mean duration of catheterization of 3.1 days, and two patients developed urinary tract infection. Altschul et al. [8] reported an infection rate of $14 \%$ in the retention group, while Hollman et al. [12] reported a rate of $1.5 \%$ infection among 150 patients with retention following joint replacement.

POUR has been reported to prolong the duration of hospital stay $[1,6]$; however, this study did not find a significant difference. This can be attributed to the fact that the routine discharge for patients undergoing microdiscectomy patients and decompressions in this study was scheduled on postoperative day 3 . The authors speculate that POUR may have a greater impact on the duration of stay, particularly, when procedures, such as microdiscectomy and lumbar decompressions, are performed on a day care basis or when the patients have a shorter postoperative stay.

Numerous studies have reported higher rates of POUR, especially in diabetics. This is believed to be attributable to neuropathy with long-term diabetes mellitus that can result in autonomic dysfunction and voiding difficulties $[1,6,9,10]$. In contrast, Altschul et al. [8] retrospectively reported on 397 elective spine surgery procedures, including cervical, thoracic, and lumbar surgical procedures to report that diabetes mellitus was not an independent risk factor. Similar findings were reported by Hollman et al. [12] based on their study on 376 patients undergoing total hip replacement surgery; they found that diabetes mellitus was not a risk factor.

\section{Conclusions}

The prevalence of POUR in posterior lumbar surgery patients was $16.5 \%$, with older age, higher BMI, longer surgery duration, higher volume of IV fluids, fusion surgery, 
and higher postoperative pain scores being significant risk factors for the development of POUR. Among them, postoperative VAS scores appeared to play an independent, strong, and significant role compared to the other factors.

\section{Conflict of Interest}

No potential conflict of interest relevant to this article was reported.

\section{Acknowledgments}

The project was funded by Ganga Orthopaedic Research \& Education Foundation, Coimbatore. The grant was received by the institute.

\section{Author Contributions}

Analysis of data and drafting of manuscript: Siddharth Narasimhan Aiyer; data collection: Ajit Kumar; conception of study and critical review of manuscript: Ajoy Prasad Shetty; drafting of manuscript: Rishi Mugesh Kanna; supervision: Shanmuganath Rajasekaran; and final approval: all authors.

\section{References}

1. Baldini G, Bagry H, Aprikian A, Carli F. Postoperative urinary retention: anesthetic and perioperative considerations. Anesthesiology 2009;110:1139-57.

2. Fernandez MA, Karthikeyan S, Wyse M, Foguet P. The incidence of postoperative urinary retention in patients undergoing elective hip and knee arthroplasty. Ann R Coll Surg Engl 2014;96:462-5.

3. Lingaraj K, Ruben M, Chan YH, Das SD. Identification of risk factors for urinary retention following total knee arthroplasty: a Singapore hospital experience. Singapore Med J 2007;48:213-6.

4. Oishi CS, Williams VJ, Hanson PB, Schneider JE, Colwell CW Jr, Walker RH. Perioperative bladder management after primary total hip arthroplasty. J Arthroplasty 1995;10:732-6.

5. O'Riordan JA, Hopkins PM, Ravenscroft A, Stevens JD. Patient-controlled analgesia and urinary retention following lower limb joint replacement: prospective audit and logistic regression analysis. Eur J Anaesthesiol 2000;17:431-5.
6. Sung KH, Lee KM, Chung CY, et al. What are the risk factors associated with urinary retention after orthopaedic surgery? Biomed Res Int 2015;2015:613216.

7. Toyonaga T, Matsushima M, Sogawa N, et al. Postoperative urinary retention after surgery for benign anorectal disease: potential risk factors and strategy for prevention. Int J Colorectal Dis 2006;21:676-82.

8. Altschul D, Kobets A, Nakhla J, et al. Postoperative urinary retention in patients undergoing elective spinal surgery. J Neurosurg Spine 2017;26:229-34.

9. Gandhi SD, Patel SA, Maltenfort M, et al. Patient and surgical factors associated with postoperative urinary retention after lumbar spine surgery. Spine (Phila Pa 1976) 2014;39:1905-9.

10. Jung HJ, Park JB, Kong CG, Kim YY, Park J, Kim JB. Postoperative urinary retention following anterior cervical spine surgery for degenerative cervical disc diseases. Clin Orthop Surg 2013;5:134-7.

11. Boulis NM, Mian FS, Rodriguez D, Cho E, Hoff JT. Urinary retention following routine neurosurgical spine procedures. Surg Neurol 2001;55:23-7.

12. Hollman F, Wolterbeek N, Veen R. Risk factors for postoperative urinary retention in men undergoing total hip arthroplasty. Orthopedics 2015;38:e507-11.

13. Wald HL, Ma A, Bratzler DW, Kramer AM. Indwelling urinary catheter use in the postoperative period: analysis of the national surgical infection prevention project data. Arch Surg 2008;143:551-7.

14. David TS, Vrahas MS. Perioperative lower urinary tract infections and deep sepsis in patients undergoing total joint arthroplasty. J Am Acad Orthop Surg 2000;8:66-74.

15. Greig JD, Mahadaven M, John TG, Garden OJ. Comparison of manual and ultrasonographic evaluation of bladder size in patients prior to laparoscopy. Surg Endosc 1996;10:432-3.

16. Griffiths CJ, Murray A, Ramsden PD. Accuracy and repeatability of bladder volume measurement using ultrasonic imaging. J Urol 1986;136:808-12.

17. Lamonerie L, Marret E, Deleuze A, Lembert N, Dupont M, Bonnet F. Prevalence of postoperative bladder distension and urinary retention detected by ultrasound measurement. Br J Anaesth 2004;92:5446. 